\title{
Molecular arrangement of a mixture of organosulfur surfactants at the aqueous solution-vapor interface studied by photoelectron intensity and angular distribution measurements and molecular dynamics simulations
}

Tanza Lewis, ${ }^{1}$ Bernd Winter, ${ }^{2 *}$ Stephan Thürmer, ${ }^{3}$ Robert Seidel, ${ }^{4}$ Anne B. Stephansen, ${ }^{2}$ J. Alfredo Freites, ${ }^{1}$ Douglas J. Tobias, ${ }^{1}$ and John C. Hemminger ${ }^{1,2 *}$

${ }^{1}$ Department of Chemistry, University of California, Irvine, California 92697, USA

${ }^{2}$ Molecular Physics Department, Fritz-Haber-Institute of the Max-Planck-Society, Faradayweg 46, D-14195 Berlin, Germany

${ }^{3}$ Department of Chemistry, Graduate School of Science, Kyoto University, KitashirakawaOiwakecho, Sakyo-Ku, Kyoto 606-8502, Japan

${ }^{4}$ Division of Renewable Energy, Helmholtz-Zentrum Berlin, Albert-Einstein-Strasse 15, D-12489 Berlin, Germany

*JCH email: jchemmin@uci.edu; *BW email: winter@fhi-berlin.mpg.de 


\begin{abstract}
Photoelectron angular distributions (PADs) from aqueous solution surfaces reveal details on the spatial arrangement of solute molecules at the solution - gas-phase interface. This is demonstrated here for mixed equimolar aqueous solutions of dimethyl sulfoxide / dimethyl sulfone $\left(\left(\mathrm{CH}_{3}\right)_{2} \mathrm{SO} /\left(\mathrm{CH}_{3}\right)_{2} \mathrm{SO}_{2}\right)$, and dimethyl sulfoxide / dimethyl sulfite $\left(\left(\mathrm{CH}_{3}\right)_{2} \mathrm{SO} /\left(\mathrm{CH}_{3}\right)_{2} \mathrm{SO}_{3}\right.$, all molecules having a propensity to reside near the solution surface. Although the surface-active molecules coexist at the surface, $\left(\mathrm{CH}_{3}\right)_{2} \mathrm{SO}_{2}$ yields a more intense sulfur $2 \mathrm{p}$ surface photoelectron signal than for $\left(\mathrm{CH}_{3}\right)_{2} \mathrm{SO}$, and for $\left.\left(\mathrm{CH}_{3}\right)_{2} \mathrm{SO}_{3}\right)$ the effect is even larger. To understand this behavior we have for one of the solutions mixtures, $\left(\mathrm{CH}_{3}\right)_{2} \mathrm{SO} /\left(\mathrm{CH}_{3}\right)_{2} \mathrm{SO}_{2}$, performed PAD measurements. Surprisingly, both molecules exhibit almost identical PADs implying that the emitted photoelectrons have experienced similar (limited) amount of scattering interactions. Hence, the molecules reside at the same distance with respect to the solution - vacuum interface rather than $\left(\mathrm{CH}_{3}\right)_{2} \mathrm{SO}_{2}$ being closer to the surface than $\left(\mathrm{CH}_{3}\right)_{2} \mathrm{SO}$, as one may have assumed based on the relative photoelectron signal intensities. Instead, the relative surface and bulk concentrations of the two compounds differ. We also report S 2p photoelectron spectra from single-component dimethyl sulfide, $\left(\mathrm{CH}_{3}\right)_{2} \mathrm{~S}$, aqueous solutions measured at a single detection angle. The exceptionally large surface propensity of $\left(\mathrm{CH}_{3}\right)_{2} \mathrm{~S}$ is recognized by a narrow, gas-phase-like photoelectron spectrum revealing that $\left(\mathrm{CH}_{3}\right)_{2} \mathrm{~S}$ experiences very little hydration interactions. Experimentally observed trends in surface activity for the different molecules, which are complemented here by molecular dynamics simulations, agree with findings obtained with other surface sensitive techniques. New information on the surface structure of mixed solutions is uniquely obtained from the anisotropic angular distributions of the photoelectrons.
\end{abstract}

\title{
I. Introduction
}

The specific properties of interfaces that cause their importance and widespread interest arise from the asymmetry in forces experienced by species at the interface. Reactivity of an aqueous solution surface, for instance of an atmospheric aerosol, with impinging gas-phase molecules is governed by molecular composition, structure, and orientation of surface molecules. Accessing such structure details from highly volatile solutions is experimentally challenging, and has typically been attempted by surface-sensitive non-linear optical spectroscopy methods including second harmonic generation ${ }^{1-8}$ (SHG) and vibrational sum frequency generation (SFG) ${ }^{9-12}$, where differentiation of surface vs. bulk species simply relies on the fact that such second-order processes are dipole forbidden in centrosymmetric media such as bulk liquids. ${ }^{4}$ Fewer studies have used surface-sensitive photoelectron (PE) spectroscopy from aqueous phase, including early 
measurements from liquid beams ${ }^{13}$ and rotating wetted disks, ${ }^{14}$ and more recent studies from a vacuum liquid microjet ${ }^{15-20}$ as well as from deliquesced salts. ${ }^{21}$ In PE spectroscopy, discrimination of surface species from species in the bulk relies on the electron kinetic energy dependent probing depth.

The different spectroscopic techniques applied to access information on surface properties probe different physical quantities, but a common goal is to quantify the amount of solute molecules adsorbed at the solution surface as a function of bulk solution concentration. If a spectroscopic signature of the adsorbate can be unequivocally identified, one can determine the surface mole fraction from which an experimental value of the Gibbs free energy for adsorption can be inferred from a Langmuir isotherm analysis. ${ }^{22-23}$ Assignment to the exact concentration of species adsorbed at the aqueous solution surface is, however, often complicated because interpretation of spectra is not straightforward. One major difficulty is to accurately determine the probing depth for a given experiment. In the case of SFG and SHG for instance, surface sensitivity refers to an interfacial region over which the inversion symmetry is broken; the length of this region is not a priori known. ${ }^{8}$ ${ }^{24}$ For PE spectroscopy, the uncertainty of probing depth is connected with the not well established inelastic mean free path of photoelectrons in solution. These issues have previously been discussed for few aqueous solution systems, including alkali halides and several atmospherically relevant surface-active molecules. ${ }^{17-18,21,25-26}$ Unlike typical PE measurements, non-linear optical studies go beyond the mere determination of surface coverage by also providing information on molecular orientation at the surface. ${ }^{4,8,27} \mathrm{PE}$ experiments on the other hand, provide a probe of the "chemical structure" of the surface, including hydration structure and ion-pairing, either via chemical shifts ${ }^{15-}$ $16,18,25,28-29$ or via probing of non-local relaxation processes. ${ }^{30-32}$

The present study explores for the first time how the photoelectron angular distributions (PAD) associated with surface-active molecules in aqueous solution provide important additional information on surface properties. We demonstrate that the PAD and the inferred angular anisotropy parameters are essential for the correct interpretation of measured PE signals with regard to how solutes accommodate at the solution surface. Our specific example, using an aqueous solution of a mixture of two different surfactant molecules, shows that only if we know the PADs can we differentiate between multiple effects that can contribute to intensity variations in the observed PE signals. Thus, from combined PAD and PE signal intensity analysis can we unequivocally identify whether solute molecules are located in the top solution layer or rather reside deeper into the solution. This is not a trivial question in a solution where two surface-active molecules compete for surface adsorption sites. From an experimental perspective, this is furthermore an important rarely considered aspect in the application of PE spectroscopy to the study 
of surface phenomena. In the current work, we focus on organosulfur aqueous solutions, as a case study, since these molecules exhibit strong surface adsorption and are of great general interest, as we discuss in the next paragraph. For the PAD experiments the use of mixed solutions is advantageous because relative electron signal intensities can be compared in the same spectrum.

Organosulfur compounds are prevalent components of atmospheric aerosols. ${ }^{33}$ The largest natural source of sulfur is dimethyl sulfide (DMS), $\left(\mathrm{CH}_{3}\right)_{2} \mathrm{~S}$, produced by phytoplankton in the ocean. ${ }^{34-35}$ DMS molecules emitted by the ocean condense into sea salt aerosols and undergo oxidation to produce dimethyl sulfoxide (DMSO), $\left(\mathrm{CH}_{3}\right)_{2} \mathrm{SO}$, dimethyl sulfone $\left(\mathrm{DMSO}_{2}\right)$, $\left(\mathrm{CH}_{3}\right)_{2} \mathrm{SO}_{2}$, dimethyl sulfite (DMSO$),\left(\mathrm{CH}_{3}\right)_{2} \mathrm{SO}_{3}$, and sulfuric acid either in the gas phase and/or at the gas - aerosol interface. ${ }^{35-37}$ The specific chemistry that occurs ultimately determines the properties of the interface. The interfacial chemical and physical properties of organosulfurcontaining aerosols are of great interest to the atmospheric chemistry community for several reasons. An obvious aspect is that aerosols scatter and absorb solar radiation; a connected aspect is that the chemical and physical properties of atmospheric particles affect the rate of aerosol formation and growth. ${ }^{38}$ As physical properties change dramatically upon oxidation, understanding the chemistry involved in atmospheric processing of e.g., DMS is essential. ${ }^{37}$ Yet, the surface propensity of aqueous DMS, arguably one of the most important compounds related to the global sulfur cycle, ${ }^{35}$ is not well understood. Also, consistent surface tension measurements could not be acquired for DMS because of its volatility. ${ }^{27}$ But the molecule is assumed to be surface active due to the hydrophobic methyl groups.

The propensity of DMSO, $\mathrm{DMSO}_{2}$, and $\mathrm{DMSO}_{3}$ for existing at the solution surface is much better characterized than the case of DMS. All three oxidized organosulfur compounds are surfaceactive, suggesting that they all play a significant role in atmospheric surface chemistry. ${ }^{37}$ SFG experiments ${ }^{27,39-40}$ have revealed an average molecular orientation with the hydrophobic methyl groups pointing away from the solution. Surface-tension measurements indicate that $\mathrm{DMSO}_{3}$ exhibits the largest surface propensity, followed by $\mathrm{DMSO}_{2}$, and lastly DMSO. ${ }^{27}$ Although DMS is believed to exhibit an even larger surface propensity, experimental measurements (both SFG and surface tension measurements) were inconclusive as aqueous DMS is highly volatile and therefore (partially) escapes the surface during measurements rendering reproducible conditions difficult. ${ }^{27}$

In the present liquid-jet PE spectroscopy study we identify each molecule by the characteristic sulfur (S) 2p electron binding energy obtained from the PE spectrum. PAD measurements, from which we infer details on the positions of solute molecules at the solution interface, are reported exemplarily for DMSO and $\mathrm{DMSO}_{2}$ aqueous solution mixtures. For equimolar DMSO / $\mathrm{DSO}_{3}$ aqueous solutions we present the PE spectrum measured at a single 
detection angle. Also, measurements on single-component DMS were conducted at a single detection angle; for aqueous DMS the interfacial structure is instead analyzed from the shape of the DMS PE spectrum, which is found to dramatically contrast the spectral shapes observed for the sulfur-oxo species. The observed trends in relative PE signal intensities of the different organosulfur molecules, connected with the qualitative difference in the surface adsorption behavior, is supported by molecular dynamics (MD) simulations.

\section{Methods}

\section{a. Experimental}

Photoelectron (PE) spectroscopy measurements were performed from a 20- $\mu \mathrm{m}$ sized liquid vacuum jet at two different undulator beamlines at the BESSY synchrotron light facility, Berlin. ${ }^{41}$ For the fixed-angle measurements performed on all four species, DMS, DMSO, $\mathrm{DMSO}_{2}$, and $\mathrm{DMSO}_{3}$ in aqueous solution we used the (former) soft-X-ray U41 PGM undulator beamline. In this case, electrons were detected normal to both the synchrotron-light polarization vector (in the floor plane) and the flow of the liquid jet. The identical setup was used for measuring the PADs, however, here the electron detection axis was varied between $0-90^{\circ}$ with respect to the light-polarization vector, which can be accurately done at the BESSY UE52-SGM1 beamline. ${ }^{42}$ Details can be found in our recent work where we have explored PADs for oxygen 1s ionization of liquid water over a large energy range. ${ }^{25}$

For all experiments, the jet velocity was approximately $50 \mathrm{~m} \cdot \mathrm{s}^{-1}$, and the jet temperature was $6^{\circ} \mathrm{C}$ before injection into the vacuum chamber. A $100-\mu \mathrm{m}$ diameter orifice that forms the entrance to the hemispherical electron energy-analyzer is at approximately $0.5 \mathrm{~mm}$ distance from the liquid jet. This is short enough to assure that detected electrons have not suffered from inelastic scattering with gas-phase water molecules around the small sized liquid jet. At operation conditions the pressure in the interaction chamber was approximately $1.5 \cdot 10^{-4}$ mbar. The energy resolution of both beamlines was better than $200 \mathrm{meV}$ at the 260-eV incident photon energy used here, and the resolution of the hemispherical energy analyzer is constant with kinetic energy (about $200 \mathrm{meV}$ at $20 \mathrm{eV}$ pass energy). The small focal size, $23 \times 12 \mu \mathrm{m}^{2}$, of the incident photon beam at the U41 beamline allows for matching of the spatial overlap with the liquid microjet, reducing the gas-phase contributions of the measured spectra to less than 5\%. At the UE52 beamline we used a similarly small illumination size by adjusting the size of the exit slit.

Most measurements in this study were conducted such that the resulting S 2p electron kinetic energy (eKE) falls in the 80 to $140 \mathrm{eV}$ range (requiring $260 \mathrm{eV}$ or 310 photon energy for 
ionization). In this eKE regime the electron inelastic mean free path is minimal, corresponding to approximately $20 \AA$ probing depth. ${ }^{17}$ The experiment thus detects PE signal from the organosulfur solution interface, referred to as a surface measurement. For the PAD measurements we applied photon energies at $310 \mathrm{eV}$, but also higher photon energies (400 eV and $640 \mathrm{eV}$ ) which probe deeper into solution, ${ }^{17,25}$ and serves here to identify the effect of electron elastic scattering in the solution on the PAD.

All solutions were prepared by dissolving the organosulphur compounds (obtained from Sigma Aldrich and used as is) in highly demineralized water followed by sonication of the mixtures. For the $\mathrm{DMSO} / \mathrm{DMSO}_{2}$ and $\mathrm{DMSO} / \mathrm{DMSO}_{3}$ mixtures equimolar concentrations of $0.5 \mathrm{M}$ (for intensity comparison) or $1 \mathrm{M}$ (for PAD experiments, only DMSO/DMSO${ }_{2}$ ) were used. In the case of DMS, the concentration was approximately 0.2 M. Due to the high volatility of DMS the actual concentration is possibly somewhat lower. In all cases salt $(0.04$ molal $\mathrm{NaCl})$ was added to the solution to minimize the streaming potential. ${ }^{43}$

\section{b. Computational}

Classical molecular dynamics (MD) simulations of aqueous DMS and DMSO were performed using a slab geometry with unit cells of $30 \AA$ x $30 \AA$ x $100 \AA$, and were replicated using three-dimensional periodic boundary conditions. Similar slab geometries have previously shown to be large enough to support stable air - water interfaces. ${ }^{29,44-45}$ The $z$ dimension of the cell was

elongated orthogonally to the liquid - vapor interface, creating a vacuum that separates the periodic images in the vertical direction. The systems simulated contained 864 water molecules and 4 molecules of DMS or DMSO, corresponding to a nominal concentration of $0.25 \mathrm{M}$. The slab simulations were performed at constant volume and a constant temperature of $300 \mathrm{~K}$ with the NAMD 2.7b1 software package. ${ }^{46}$ The lengths of the trajectories for the DMS and DMSO slab systems were 30 and 50 ns, respectively; the first 5 ns of each trajectory was considered as equilibration and was discarded for the analysis.

To characterize the bulk solvation of DMS and DMSO, and compare it to the solvation of a nonpolar molecule of roughly the same size, MD simulations were carried out for a single DMS, DMSO, or propane molecule in bulk aqueous solution at a constant temperature of $300 \mathrm{~K}$ and a constant pressure of $1 \mathrm{~atm}$. with isotropic volume fluctuations. The bulk solution simulations of DMS, DMSO, and propane contained 401, 308, and 401 water molecules, respectively. The lengths of the trajectories for the DMS, DMSO, and propane systems were all 20 ns; the first 5 ns of each trajectory was considered as equilibration and was discarded for the analysis. 
The force field for DMS was obtained by minor modification of the CHARMM22 force field for ethylmethylsulfide, ${ }^{47}$ a model compound for the side chain in the amino acid methionine. For DMSO we used the force field developed by Stradler and Feller. ${ }^{48}$ The CHARMM36 force field was used for propane, ${ }^{49}$ and the TIP3P model was used for water. ${ }^{50}$ The equations of motion were integrated using a multiple time step algorithm ${ }^{51}$ with a time step of 4 fs for electrostatic forces, 2 fs for short-ranged non-bonded forces, and 1 fs for bonded forces. The lengths of bonds containing hydrogen atoms were constrained using the SHAKE ${ }^{52}$ and SETTLE ${ }^{53}$ algorithms. The smooth particle mesh Ewald method ${ }^{54}$ with a $4^{\text {th }}$ order interpolation was used to calculate the electrostatic energies and forces, and the short-range, real-space interactions were smoothly truncated at $11 \AA$ using a switching function. The temperature was controlled using Langevin dynamics with a damping coefficient of $1 \mathrm{ps}^{-1}$. In the constant pressure, bulk simulations, the pressure was controlled using a Nose-Hoover-Langevin piston. ${ }^{55-56}$ The VMD software package ${ }^{57}$, version 1.9.3, was used for the molecular graphics.

\section{Results and Discussion}

\section{a. Trends in electron binding energies and signal intensities}

Figure 1 presents S 2p PE spectra of mixed equimolar (0.5 M) solutions of aqueous DMSO / $\mathrm{DMSO}_{2}$ (top figure) and DMSO / $\mathrm{DMSO}_{3}$ (bottom figure); the spectra illustrate that the different molecules can be distinguished by their respective electron binding energies (BE). PE spectra from DMS (aq) will be considered separately as DMS exhibits a very different spectral shape. The S 2p PE spectra in Figure 1 were recorded using $260 \mathrm{eV}$ photon energy thus yielding eKEs of approximately 80-90 eV corresponding to probing of the surface region. ${ }^{17} \mathrm{BEs}$ were calibrated with reference to the $1 b_{1}$ water valence energy of liquid water. ${ }^{58}$ Experimental data is presented by the dots, and grey dashed lines are Gaussian fits to the signal contributions from the respective species; DMSO (blue filling), $\mathrm{DMSO}_{2}$ (orange filling), and $\mathrm{DMSO}_{3}$ (green filling), and the red curve is the summed fit. The fits reproduce the experimental spectra well. The double-peak structure of each component (described here by two Gaussians with 1:2 peak area) is characteristic for the p-orbital spin-orbit split.

From Figure 1 we find that aqueous-phase DMSO has the lowest S 2p BEs of $173 \mathrm{eV}$ for S $2 \mathrm{p}_{3 / 2}$ and $171.9 \mathrm{eV}$ for $\mathrm{S} 2 \mathrm{p}_{1 / 2}$; the full width at half maximum (fwhm) is fitted to $1.06 \mathrm{eV}$ for both components. The observed spin-orbit peak splitting is similar to the splitting observed for the other compounds investigated herein and is also consistent with reports from literature $\left(\Delta \mathrm{E}_{\text {soc }} \approx 1.1 \mathrm{eV}^{59-}\right.$ ${ }^{61}$ ). The S 2p binding energies of $\mathrm{DMSO}_{2}(\mathrm{aq})$ and $\mathrm{DMSO}_{3}$ (aq) are both approximately $2 \mathrm{eV}$ larger than for DMSO (aq), while peak widths are the same within $0.02 \mathrm{eV}$ tolerance. Observed trends in 
BEs, the chemical shifts, can be attributed to primarily result from the specific chemical bonds of the central sulfur atom and the associated variation in oxidation, which result in different electronegativities at the sulfur site. BE differences due to variations in hydration are much smaller and cannot be quantified here; we will return to the questions of hydration energies in section III.c. Low BEs in Figure 1 can thus be associated with larger charge density at the sulfur atom; this value will decrease with the number of (electron pulling) bonds between sulfur and oxygen atoms. The similar S 2p BEs for $\mathrm{DMSO}_{2}$ and $\mathrm{DMSO}_{3}$ despite the different oxidations states ( +2 and +4 respectively) could indicate that the effect of electron-pulling by the oxygen atoms is counter-balanced (yielding similar electronegativity) upon substitution of one S-O bond by two S-C bonds. On the other hand, $\mathrm{DMSO}_{2}$ and $\mathrm{DMSO}_{3}$ effectively have the same number of $\mathrm{S}-\mathrm{O}$ bonds (albeit in different combinations of $\sigma$ and $\pi$ bonds) thus also rationalizing the similar BEs.

Concerning the key question of surface activity, the most striking observation from Figure 1 is the non-stoichiometric signal intensity ratios. Despite the equimolar concentrations the S 2p PE signal intensity ratios $I\left(\mathrm{DMSO}_{2}\right)$ / I(DMSO) and $I\left(\mathrm{DMSO}_{3}\right) / I(\mathrm{DMSO})$ are both much larger than 1. For $I\left(\mathrm{DMSO}_{2}\right) / I(\mathrm{DMSO})$ the observed ratio is approximately 2, and for $I\left(\mathrm{DMSO}_{3}\right) /$ I(DMSO) the ratio is nearly 6. This trend would seem qualitatively consistent with the aforementioned trend in surface activity, DMSO $<\mathrm{DMSO}_{2}<\mathrm{DMSO}_{3}$, as also found in ref. ${ }^{27}$. Yet, in the absence of further experimental information, surface-structure details for these mixed solutions are impossible to infer. Note that the cross sections for $\mathrm{S} 2 \mathrm{p}$ photoionization ( $\sigma_{\text {ionization }}(h v)$ in Eq. 1 of section III.b) are assumed to be the same for $\mathrm{DMSO}_{2}$ and $\mathrm{DMSO}$, and are extremely unlikely to cause such large intensity variations. Potential variations in the photoionization cross section, e.g., due to EXAFS-like (Extended X-ray Absorption Fine Structure) modulations, ${ }^{62}$ can reasonably be assumed to be negligibly small as the photon energies employed are significantly above the absorption threshold (by $100 \mathrm{eV}$ ), and, if present at all, should not be too different for both species. One would be tempted to interpret $I\left(\mathrm{DMSO}_{2}\right) / I(\mathrm{DMSO})=2$ as resulting from a layered density profile with $\mathrm{DMSO}_{2}$ residing at the top surface, while DMSO is located slightly deeper in the solution. But another possibility is that the different molecules have a similar density profile, just differing by the relative number of molecules at a given distance from the top surface. In the case of alkali-halide aqueous solutions a layered arrangement is common; in that case the density profiles of large and strongly polarizable anions ( $\mathrm{I}^{-}$in particular) peak within the first few $\AA$ below the water surface, while the density profiles of cations typically exhibit a maximum near $6 \AA$ into the solution ${ }^{17,63-65}$ (except for the case of surface active $\mathrm{Li}^{+66}$ ).

In the present case, where both molecules coexisting in solution are surface-active, such a picture might not be applicable, and we may rather expect both species to preferentially localize at 
the interface and exhibit density profiles peaking at the same distance from the surface. The species with the larger Gibbs free energy for adsorption will however saturate the surface at lower bulksolution concentration. ${ }^{27}$ The other species (with lower Gibbs free energy for adsorption) would saturate the surface at relatively higher concentration, and the density profile would extend further into the bulk solution.

This would lead to the same qualitative trend of relative intensities observed in Figure 1. In Figure 2 we show cartoon pictures of these two possible density profiles. At the top of Figure 2 we illustrate a simple layered profile. At the bottom of Figure 2 a different profile is shown in which the density profiles of the two solutes peak at the same distance from the surface but the density profile of one of the solutes (in this case DMSO) extends more into the bulk of the solution. Simply looking at the photoelectron intensity data does not allow us to differentiate between these two situations. We show below that measurements of the photoelectron angular distributions provide evidence that the density profiles are more accurately described by the lower picture of Figure 2 . However, before we discuss this important issue, which has significant consequences for PE spectroscopy depth-profile determinations of liquid solution in general, we address the behavior of the highly volatile DMS molecule at the aqueous solution surface.

Figure 3 shows the S 2p PE spectrum of aqueous DMS at a concentration of $0.2 \mathrm{M}$ which is close to the maximum solubility. The photon energy was again $260 \mathrm{eV}$, i.e., within the surface sensitive regime. As in the case of the oxo-sulfur compounds, the spectrum of DMS is fit by two Gaussians yielding S 2 $\mathrm{p}_{3 / 2}$ and S 2 $\mathrm{p}_{1 / 2}$ BEs of $169.0 \mathrm{eV}$ and $170.1 \mathrm{eV}$, respectively. This is lower than the BEs of the oxidized compounds by roughly $2.9 \mathrm{eV}$ and $4.9 \mathrm{eV}$ (Figure 1). Using the same argument as above, the lower S 2p BE of DMS can be attributed to be mainly due to larger electron density at the sulfur site compared to the other molecules studied here, which again is consistent with the lowest oxidation state associated with the sulfur atom (-2 for DMS). A notable feature of Figure 3, contrasting the spectra of Figure 1, is the narrow widths of the S 2p PE spectrum (fwhm $=0.5 \mathrm{eV}$, i.e., ca. $0.6 \mathrm{eV}$ less than observed for all other compounds investigated here). The narrow peak widths essentially yield baseline resolution between the two spin-orbit peaks. Such a narrow peak width is unusual in liquid-phase spectroscopy, where usually peak width of $>1 \mathrm{eV}$ are observed which reflect the many different hydration configurations, ${ }^{67}$ but is typical for gasphase/non-hydrated species. Since the experimental conditions for all measurements reported herein stay unchanged when injecting different solutions, we always probe the same part of the liquid microjet, and we probe equally deep into solution. Also, thermodynamic (local) equilibration between the liquid-jet surface and the vapor in our experiment ${ }^{18}$ assures detection of both gas-phase and surface-bound molecules. ${ }^{17}$ Our data thus implies that DMS molecules are less hydrated at the 
solution surface than the sulfur-oxo counterparts. Being significantly exposed to the vapor side and simultaneously easily evaporating (compare Introduction), the hydration number of DMS must be small. This interpretation of our observations is consistent with vibrational SFG studies concluding that the DMS - water interactions are weakest of all adsorbates considered here. Moreover, strong exclusion of DMS molecules from the bulk is also supported by our MD simulations, which will be discussed in section III.c.

\section{b. Photoelectron angular distributions as probe for solute arrangements}

We return to the question of how DMSO and $\mathrm{DMSO}_{2}$ molecules are accommodated at the solution interface by invoking PAD measurements. Any anisotropic PAD, characteristic of ionization from a given orbital of a gas-phase molecule, may be altered by two possible contributions when measured from aqueous-phase: (i) Alteration of the orbital character by intermolecular interactions and (ii) scattering of the outgoing electron within the liquid. As the $\mathrm{S}$ $2 p$ core-level orbitals of the organosulfur solutes probed here do not engage in intermolecular interactions with the surroundings, the primary source for variations in the PADs is expected to result from scattering interactions. Any anisotropic PAD will smear out when measured in aqueous solution due to random elastic scattering of the photoelectrons; ${ }^{17}$ i.e., the anisotropy will decrease. The PAD is described in the framework of the differential cross section for ionization of randomly oriented molecules, when using linearly polarized light:

$$
\frac{d \sigma_{\text {ionization }}(E)}{d \Omega(\theta)}=\frac{\sigma_{\text {ionization }}(E)}{4 \pi}\left[1+\beta_{2}(E) P_{2}(\cos (\theta))+\cdots\right]
$$

In this expression $\sigma_{\text {ionization }}(\mathrm{h} v)$ is the total photoionization cross section, $\Omega$ is the solid angle of detection, $P_{2}(x)$ is the 2nd order Legendre polynomial, and $\theta$ the detection angle measured with respect to the polarization vector of the incident light. Here, the anisotropy parameter, $\beta_{2}(E)$, which depends on the sub-shell of origin and the eKE, ${ }^{17,69}$ is the sole parameter determining the PAD, and can be obtained from fitting the angle-dependent experimental PE spectra. Higher order or odd terms are typically only included when multiphoton processes or circular light polarization are used in the photoionization process, but they are irrelevant here. Since $d \sigma_{\text {ion }} / d \Omega$ must be positive, $\beta_{2}$ ranges from -1 to 2 , for $\beta_{2}=0$ the photoemission is isotropic.

Importantly, for the application of Eq. 1 on liquid-phase molecules, the effect of scattering interactions on the measured PADs is strongest when the cross section for elastic scattering is larger than the one for inelastic scattering. This is the case for eKEs below approximately $100 \mathrm{eV}$ in liquid 
water as demonstrated in ref. ${ }^{25}$. In terms of electron attenuation lengths or probing depth (which is the more useful description in PE spectroscopy), a larger electron probing depth leads to larger smear-out of the liquid-phase $\mathrm{PAD}$ relative to the respective gas-phase $\mathrm{PAD}$, and thus smaller values of $\beta_{2}$ (loss of anisotropy). For ionization of oxygen 1s of liquid water with photon energies sufficiently above the ionization threshold (at approximately eKE $>100 \mathrm{eV}$ ), $\beta_{2}$ was found to decrease by approximately $20 \%$ relative to the gas-phase value. Similar to the gas-phase $\beta_{2}$-value, the liquid-phase $\beta_{2}$-value levels off asymptotically with increasing eKE, and in the eKE $>100 \mathrm{eV}$ regime, the oxygen 1 s of liquid water is still remarkably anisotropic. ${ }^{25}$ This important result should motivate inclusion of PADs in future liquid-jet PE spectroscopy studies.

Figure 4 presents S 2p PE spectra of a $1 \mathrm{M}$ DMSO / 1M DMSO ${ }_{2}$ aqueous solution mixture similar to Figure 1A, but this time spectra are also shown for parallel $\left(\theta=0^{\circ}\right)$ and magic angle $\left(\theta=54.7^{\circ}\right)$ detection with respect to the light polarization vector. The use of $1 \mathrm{M}$ concentrations here is simply to obtain large enough PE signal intensities over the entire $0-90^{\circ}$ angle range. The spectra shown in Figure 4 were recorded at $h v=310 \mathrm{eV}$ thus yielding eKEs of 134-140 eV, i.e., well above $100 \mathrm{eV}$ but within the surface-sensitive probing range. ${ }^{17}$ We find that the total S 2p PE intensity drops uniformly by more than a factor five when going from normal to parallel detection. Notably, the PE signal intensity decreases by exactly the same factor for both molecules which is indicative of a specific common value of $\beta_{2}$. The spectral resemblance at all angles can be more clearly seen from the inset of Figure 4 showing the three spectra scaled to the same maximum intensity.

Systematic investigation of the angle-dependent signal intensities of the equimolar DMSO / $\mathrm{DMSO}_{2}$ solution indeed verifies that the $\beta_{2}$ values associated with $\mathrm{S} 2 \mathrm{p}$ ionization of both molecules are equivalent. We recorded the change of S 2p PE signal intensities (integrated peak areas) at several detection angles between $0^{\circ}$ to $90^{\circ}$; Figure 5 shows representative data obtained for aqueous $\mathrm{DMSO}_{2}$ using $310 \mathrm{eV}$ photon energy, including a fit of the experimental data to Eq. 1 . From the fit in Figure 5 we find $\beta_{2}=1.03 \pm 0.02$ for aqueous $\mathrm{DMSO}_{2}$. The corresponding value for DMSO at $h v=310$ is $\beta_{2}=1.05 \pm 0.02$ as shown in the inset of Figure 5 . The inset furthermore presents the $\beta_{2}$ values for both molecules at two additional photon energies, $h v=400 \mathrm{eV}$ and 640 $\mathrm{eV}$, yielding eKEs of $c a .230 \mathrm{eV}$ and $470 \mathrm{eV}$. It is worthwhile to mention that for the cylindrical geometry of our target, the liquid microjet, effects of molecular orientation at the solution surface average out. ${ }^{17}$ As can be seen in the inset of Figure 5, the $\beta_{2}$ values increase slightly with eKE, but the anisotropy essentially remains the same for the two solutes, clearly revealing non-isotropic PADs. In other words, information on orbital symmetry is maintained in the detection and not lost due to scattering interactions. Interestingly, the $\beta_{2}$ values for S $2 \mathrm{p}$ of aqueous $\mathrm{DMSO}$ and $\mathrm{DMSO}_{2}$ 
reported here are similar to the values reported for $\mathrm{S} 2 \mathrm{p}$ of gas-phase $\mathrm{H}_{2} \mathrm{~S}$ ( $\beta=1.1$ for approximately $90 \mathrm{eV}$ kinetic energy), argued to resemble atomic $S,{ }^{70-71}$ and even to $\mathrm{OCS}^{70,72}$, exhibiting a different molecular geometry around the sulphur atom. Hence, the reduction of $\beta$ by at most $5 \%$ measured in the present study is much less than in water, when going from gas to liquid phase. This is the expected behavior for surface-active molecules.

The implications of equivalent $\beta_{2}$ values for DMSO and $\mathrm{DMSO}_{2}$ in the mixed solution are twofold. First, the S 2p orbital structure of the two molecules must be almost the same. This may at first glance seem surprising given the approximately $2 \mathrm{eV}$ chemical shift in the $\mathrm{S} 2 \mathrm{p}$ PE spectra (compare Figure 1A). But the energy shift is likely due to aforementioned change in electronegativity, and does not necessarily translate into a significant change in inner-shell orbital structure. This notion is supported by the above mentioned similar $\beta_{2}$ values reported for $\mathrm{H}_{2} \mathrm{~S}$ and OCS. ${ }^{70-72}$ The second and more important implication is that similar amount of elastic scattering must occur for the photoelectrons emitted from DMSO and from $\mathrm{DMSO}_{2}$. Otherwise, larger variations in $\beta_{2}$ would be observed (particularly at low eKEs), characteristic for electron scattering from neat liquid water ( $\beta$ reduction of approximately 20\%). Hence both molecules must have the highest number densities at nearly the same distance from the surface.

Taken together our PAD analysis shows that both DMSO and $\mathrm{DMSO}_{2}$, also in mixed solutions, adsorb at the aqueous solution surface. Both molecules assume positions (maximum densities) at ca. the same distance from the solution surface - i.e., not a layered arrangement as evidenced by the identical $\beta_{2}$ values. The signal intensity distribution of the spectrum shown in Figure 1A, indicating approximately twice as large surface concentration of $\mathrm{DMSO}_{2}$ for the equimolar DMSO / $\mathrm{DMSO}_{2}$ solution, thereby reflects the larger adsorption energy of $\mathrm{DMSO}_{2}$ as compared to DMSO. This is consistent with the respective differences in surface concentrations as a function of DMSO and $\mathrm{DMSO}_{2}$ bulk concentration found in SFG studies. ${ }^{27}$ The few concentrations measured in the current liquid-jet PE spectroscopy experiments however do not allow for a reliable estimate of the Gibbs free energy of adsorption. Nonetheless, when combining the information obtained from our $\mathrm{PAD}$ and signal-intensity analysis of solute mixtures, a qualitative understanding of the surface structure can be inferred as graphically summarized in the cartoon presented in Figure 2. The top scenario shows the simple interpretation one might immediately (and erroneously) deduce from the intensity distributions, the bottom scenario boxed by a red square, summarizes the picture reached when taking both PAD and intensity distributions into account.

\section{c. MD simulations}


Finally, we turn to the MD simulations and assess how the theoretical results compare with the interpretation sketched in Figure 2 and additional experimental results. The experimental findings on the weakly hydrated surface DMS and the contrast to the findings on DMSO are well supported by the MD simulations. Simulations of interfacial systems were only performed for DMS and DMSO and, as such, we cannot make a direct comparison with regards to the distance of DMSO and $\mathrm{DMSO}_{2}$ from the solution surface, which we argued above to be similar (cf. Figure 2).

Liquid-vapor interfaces of DMS and DMSO aqueous solutions were simulated using a slab geometry that contained 864 water molecules and four molecules of DMS or DMSO, corresponding to a nominal concentration of $0.25 \mathrm{M}$. Figure $6 \mathrm{~A}$ and $6 \mathrm{C}$ show a representative molecular snapshot and the density profiles of the DMS S atoms and the water $\mathrm{O}$ atoms (note that $z=0$ corresponds to the center of the slab) respectively. The immediate observation from the density profiles is that DMS molecules on average reside on the solution surface, as was suggested based on the width analysis of the PE spectra (Figure 3) in section III.a. In the snapshot shown in in the upper left panel of Figure 6, one of the DMS molecules has left the water surface and two additional molecules appear to be departing, thereby indicating the presence of gas-phase DMS in the simulations. This is consistent with the very large Henry volatility of DMS. ${ }^{37,73}$ The Henry's Law constant can be calculated in units of $\mathrm{M} \cdot \mathrm{atm}^{-1}$ from the density profile for the $\mathrm{S}$ atom in DMS according to Eq. 2:

$$
k_{H}=\frac{1}{R T}\left(\frac{\rho_{l}}{\rho_{g}}\right)
$$

where $\rho_{l}$ is the average density in the liquid phase $(|z|<8 \AA), \rho_{g}$ is the average density in the gas phase $(|z|>24 \AA), R$ is the gas constant $\left(0.08206 \mathrm{~L} \cdot \mathrm{atm} \cdot \mathrm{mol}^{-1} \cdot \mathrm{K}^{-1}\right)$, and $T$ is the temperature. The value we obtain, $1.2 \times 10^{-2} \mathrm{M} \cdot \mathrm{atm}^{-1}$, is within the range of experimental values, $0.16-8.0 \times 10^{-2}$ $\mathrm{M} \cdot \mathrm{atm}^{-1}$, tabulated by Sander, ${ }^{74}$ and thus provides support for the validity of the DMS force field used here. In contrast to the extreme surface propensity of DMS, DMSO exhibits more moderate preference for the solution surface, as can be seen in the bottom right panel of Figure 6. Our MD simulations indicate that the majority of DMSO molecules reside at the surface, but migration into the bulk is also observed, as can be seen in the right two panels of Figure 6. The lower PE signal intensities of DMSO as compared to $\mathrm{DMSO}_{2}$ and $\mathrm{DMSO}_{3}$ observed in our experimental data also indicate a "moderate" surface preference of DMSO, consistent with the hygroscopic nature of DMSO. $^{27}$

Differences in the solvation characteristics of DMS and DMSO can be quantified by calculating the radial distribution functions (RDFs) of water oxygen atoms around the sulfur atoms 
of each molecule (which both represent the molecular centers and the comparable point of all investigated solutes). The RDFs for DMSO in bulk and interfacial configurations are shown in Figure 7B. For water around the S atoms in DMSO, the RDFs are well structured with a clear minimum at $4.3 \AA$, indicating strong interactions between DMSO and solvating water molecules. The significant hydration of DMSO is experimentally evident from the substantial peak widths of the associated PE spectra (Figure $1, \mathrm{fwhm} \approx 1.06 \mathrm{eV}$ ). In contrast, the RDFs for water around the $\mathrm{S}$ atom in DMS are less structured both at the aqueous interface and in bulk solution (Figure 7A), and the structuring of water around DMS is intermediate between that of water around DMSO (Figure $7 \mathrm{~B}$ ) and the central $\mathrm{C}$ atom $\left(\mathrm{C}_{2}\right)$ of the non-polar molecule propane (Figure $7 \mathrm{C}$ ). The latter observation is corroborated by the water density isosurfaces shown in Figure 8.

Additional insight into the nature of the solvation is provided by the Errington-Debenedetti tetrahedral order parameter, ${ }^{75}$ defined in Eq. 3:

$$
q=1-\frac{3}{8} \sum_{j=1}^{3} \sum_{k=j+1}^{4}\left(\cos \psi_{j k}+\frac{1}{3}\right)^{2}
$$

Here $j$ and $k$ are indices of a water $\mathrm{O}$ atom in the first solvation shell of an atom under consideration and its three nearest (water $\mathrm{O}$ ) neighbors, and $\psi_{j k}$ is the angle between lines connecting the atom under consideration with its four nearest water $\mathrm{O}$ atoms. Note that $q$ is equal to zero for an ideal gas and one for perfect tetrahedral order. Histograms of $q$ averaged over all of the water $\mathrm{O}$ atoms in the first solvation shell (defined as water $\mathrm{O}$ atoms whose distance from the atom under consideration is less than the position of the first minimum in the RDF) around other water $\mathrm{O}$ atoms, the $\mathrm{S}$ atom in DMS, the S atom in DMSO, and the central carbon in propane in bulk water are plotted in Figure 9; the corresponding average values of $\langle q\rangle$ are 0.56, 0.51, 0.47, and 0.54, respectively. Consistent with a previous simulation study of the hydration of the alkyl chain of 1propanol in bulk water under ambient temperature and pressure, ${ }^{76}$ the tetrahedral order of water solvating the hydrophobic propane molecule is only very slightly lower (0.02 on average) than that of water solvating a water molecule. Even at high pressure (30 MPa), the reduction of the tetrahedral order by the alkyl chain of 1-propanol is small ( 0.05) at $300 \mathrm{~K} .{ }^{77}$ DMSO causes the greatest distortion of the tetrahedral ordering of its hydration shell among the molecules considered and, consistent with the other hydration characteristics reported above (RDFs and isodensity surfaces), the effect of DMS on the tetrahedral order is intermediate between the polar molecule DMSO and the non-polar molecule propane. 
Calculated coordination numbers in the first solvation shell (defined using $4.3 \AA$ cutoff radius, which is the position of the first minimum in the $\mathrm{RDF}$ for water $\mathrm{O}$ atoms around DMS or DMSO S atoms) of DMS and DMSO are presented in Figure 10 as functions of the position relative to the Gibbs dividing surface or GDS (the GDS is the position along the normal to the interface where the water density is equal to half its bulk value). The number of water molecules hydrating DMS is found to be smaller by $0.5-1.7$ on average compared to DMSO, throughout the interfacial region and into the bulk.

\section{d. Binding energy shifts}

The analysis of the solvation of DMS and DMSO in the MD simulations revealed that interactions between DMS and water molecules are relatively weak compared to DMSO. This is consistent with the narrow fwhm of the PE features of DMS of ca. $0.5 \mathrm{eV}$ (Figure 3). Concluding our comparative analysis of the MD simulations and the PE experiments, we briefly return to the BE shifts observed in Figure 1 and 3 as a function of the structure of the organosulfur compounds. Experimental BE shifts and bonding motifs on the sulfur atom are summarized in Table I. Interestingly, it appears that each additional pair of bonds to oxygen leads to an increase in the $S$ 2p binding energy of 2-3 eV. In section III.a. changes in BE were argued to mainly result from the specific chemical bonds to the center $\mathrm{S}$ atom, and other effects were argued to be comparatively negligibly small. This notion is explored in some further detail here. More generally speaking, shifts in BE may result from chemical shifts, changes in surface dipoles, electronic polarization, and/or changes in orbital structure due to specific intermolecular interactions. ${ }^{63,67}$ The effect of surface dipoles is typically small, for oriented water molecules the effect only assumes a value of $\approx 10 \mathrm{mV}^{67,78}$ and we can thus to a reasonable approximation neglect that contribution here. From our analysis above along with the results of Tarbuck et al. ${ }^{27}$ changes in orbital structure due to specific intermolecular interactions should also be negligible. Furthermore, from a structural perspective such effects should primarily affect oxygen and not $\mathrm{S}$. When specific solvation effects are negligible, the effect of solvation on the BE is mainly due to electronic polarization. The remaining contributions to consider are thus the chemical shift and the effect of solvation by electronic polarization, which we consider using two different approaches below.

The PE spectra of all oxo-sulfur compounds showed fwhms of roughly $1 \mathrm{eV}$ contrasting the fwhm observed in the PE spectrum (Figure 3) of DMS of ca $0.5 \mathrm{eV}$, which in the discussion above, was taken as an indication of different extents of hydration, with DMS being least solvated. This interpretation was supported by the MD simulations on DMS and DMSO. Furthermore, the PAD analysis indicates that the majority of the DMSO and $\mathrm{DMSO}_{2}$ molecules reside at a similar 
distance from the surface. These combined observations suggest that the effect of hydration on the BEs is similar for $\mathrm{DMSO}, \mathrm{DMSO}_{2}$, and $\mathrm{DMSO}_{3}$, but significantly different for DMS. This notion enables separation (albeit in a somewhat approximate manner) of the energy shift associated with chemical bonding to $\mathrm{S}$ from the effect of solvation. When assuming the energy stabilization associated with solvation is similar for $\mathrm{DMSO}, \mathrm{DMSO}_{2}$, and $\mathrm{DMSO}_{3}$, the energy shifts summarized in Table I suggest that two additional S-O bonds result in a BE shift of ca. $2 \mathrm{eV}(=4.9 \mathrm{eV}-2.9$ $\mathrm{eV}$ ). The remaining change in BE when going from DMS to DMSO (which also involves a change of two S-O bonds) is approximately $0.9 \mathrm{eV}=(2.9 \mathrm{eV}-2 \mathrm{eV})$. From the above arguments this should (very) roughly compare to the additional stabilization energy due to solvation that all oxosulfur compounds experience as compared to DMS.

When specific solvation effects can be neglected, the effect of solvation can also be estimated from the Born equation for the Gibbs free energy of solvation ${ }^{67}$

$$
\Delta G=-\frac{e^{2} q^{2}}{8 \pi \epsilon_{0} R_{e f f}}\left(1-\frac{1}{\epsilon}\right)
$$

Since photoemission is much faster than solvent reorientation, the relative permittivity, $\epsilon$, can be approximated to the optical macroscopic permittivity of water, $\epsilon_{o p t}=1.8$, and $R_{e f f}$ refers to the first maximum of the water-molecule RDF. ${ }^{67}$ As the RDF of water-DMS (Figure 7A) is structureless, we can only make a reasonable estimate of the electronic polarization energy for DMSO. As can be seen from Figure 7B, the first maximum in the RDF for water-DMSO is at ca. $3.5 \AA$. Using

Eq. 4. this $R_{e f f}$ value yields a $\Delta G$ of $\approx 0.9 \mathrm{eV}$, i.e. the same energy as was obtained in our first approach to evaluate the difference in solvation energy. Albeit very approximate, this notion is consistent with the overall analysis, and supports the interpretation that DMS experiences negligible stabilization via solvation interactions, even when residing on/near the water surface. The equal values of solvation reached via the two different approaches also indicate that the estimate of the chemical shifts associated with additional bonding to oxygen is reasonable. The differences in hydration between the oxo-sulfur compounds are however more delicate, and cannot be assessed with the approximate models employed here.

\section{Conclusions}

In conclusion, we have presented liquid-jet S 2p PE spectroscopy studies from mixed DMSO / $\mathrm{DMSO}_{2}$ and DMSO / $\mathrm{DMSO}_{3}$ aqueous solutions, and from single-component DMS aqueous solution. For the DMSO / $\mathrm{DMSO}_{2}$ system the photoelectron angular 
distributions of both molecular components were measured. The different surface propensities of the organosulfur molecules are consistent with our MD simulations. The key findings can be summarized as

(1) Signal intensity distributions of the oxo-sulfur species indicate that $\mathrm{DMSO}_{3}$ occupies more surface sites than $\mathrm{DMSO}_{2}$, and both molecules occupy more sites than DMSO in the respective equimolar mixtures. Yet all three molecules exhibit high surface propensity.

(2) The PAD analysis of the PE data recorded for the $\mathrm{DMSO} / \mathrm{DMSO}_{2}$ mixture reveal that the surface prone molecules arrange in a mixed structure as opposed to a layered arrangement. This interpretation was derived from the similar $\beta_{2}$-values observed for the PADs of DMSO and $\mathrm{DMSO}_{2}$ indicating that the photoelectrons experience some elastic scattering within the interfacial region, before reaching the detector.

(3) The PE spectrum of DMS (aq) is considerably narrower than the spectra of the oxospecies. In agreement with MD simulations and the magnitude of the observed BE shifts this behavior is attributed to the very weak interaction between DMS and water resulting in an exceptionally large surface propensity of DMS.

The impact of our results is twofold: Regarding the interfacial structure revealed here, we have demonstrated that in the DMSO / $\mathrm{DMSO}_{2}$ mixed aqueous solution both molecules reside near the surface and can therefore participate in immediate chemical reactions with impinging gas-phase molecules. From a spectroscopic perspective, we have demonstrated the importance of considering angular information in the interpretation of relative PE signal intensities, and how intensity distributions translate to surface structure. This is a highly important argument for future applications of PE spectroscopy to the study of interfacial physics and chemistry.

\section{Acknowledgments}

AirUCI (an organized research unit at the University of California, Irvine) under Grant CHE 0431312 from the NSF supported this work. B.W. acknowledges support from the Deutsche Forschungsgemeinschaft (DFG) via the DFG Research Unit FOR 1789. B.W. and R.S. furthermore acknowledge support from the DFG through the Collaborative Research Center 1109. Liquid-jet photoelectron spectroscopy experiments were carried out at the BESSY Synchrotron, HelmholtzZentrum Berlin. 
1. Eisenthal, K. B., Photochemistry and Photophysics of Liquid Interfaces by Second Harmonic Spectroscopy. J. Phys. Chem. 1996, 100, 12997-13006.

2. Wang, H.; Yan, E. C. Y.; Borguet, E.; Eisenthal, K. B., Second Harmonic Generation from the Surface of Centrosymmetric Particles in Bulk Solution. Chem. Phys. Lett. 1996, 259, 15-20.

3. Shi, X.; Borguet, E.; Tarnovsky, A. N.; Eisenthal, K. B., Ultrafast Dynamics and Structure at Aqueous Interfaces by Second Harmonic Generation. Chem. Phys. 1996, 205, 167-178.

4. Eisenthal, K. B., Liquid Interfaces Probed by Second-Harmonic and SumFrequency Spectroscopy. Chem. Rev. 1996, 96, 1343-1360.

5. $\quad$ Petersen, P. B.; Johnson, J. C.; Knutsen, K. P.; Saykally, R. J., Direct Experimental Validation of the Jones-Ray Effect. Chem. Phys. Lett. 2004, 397, 46-50.

6. Petersen, P. B.; Saykally, R. J., Confirmation of Enhanced Anion Concentration at the Liquid Water Surface. Chem. Phys. Lett. 2004, 397, 51-55.

7. Heinz, T. F.; Tom, H. W. K.; Shen, Y. R., Determination of Molecular-Orientation of Monolayer Adsorbates by Optical 2nd-Harmonic Generation. Phys.l Rev. A 1983, 28, 1883-1885.

8. Shen, Y. R., Surface-Properties Probed by 2nd-Harmonic and Sum-Frequency Generation. Nature 1989, 337, 519-525.

9. Zhu, X. D.; Suhr, H.; Shen, Y. R., Surface Vibrational Spectroscopy by InfraredVisible Sum Frequency Generation. Phys. Rev. B 1987, 35, 3047-3050.

10. Zhang, Z.; Guo, Y.; Lu, Z.; Velarde, L.; Wang, H.-f., Resolving Two Closely Overlapping -Cn Vibrations and Structure in the Langmuir Mono Layer of the LongChain Nonadecanenitrile by Polarization Sum Frequency Generation Vibrational Spectroscopy. J. Phys. Chem. C 2012, 116, 2976-2987.

11. Zhang, D.; Gutow, J. H.; Eisenthal, K. B., Structural Phase Transitions of Small Molecules at Air/Water Interfaces. J. Chem. Soc., Faraday Trans. 1996, 92, 539-543.

12. Zhang, D.; Gutow, J. H.; Eisenthal, K. B.; Heinz, T. F., Sudden Structural-Change at an Air Binary-Liquid Interface - Sum Frequency Study of the Air Acetonitrile-Water Interface. J. Chem. Phys. 1993, 98, 5099-5101.

13. Siegbahn, H.; Siegbahn, K., Esca Applied to Liquids. J. Electron Spectrosc. 1973, 2, 319-325.

14. Siegbahn, K., Nobel Lecture, Electron Spectroscopy for Atoms, Molecules and Condensed Matter, 1981.

15. Brown, M. A.; Winter, B.; Faubel, M.; Hemminger, J. C., Spatial Distribution of Nitrate and Nitrite Anions at the Liquid/Vapor Interface of Aqueous Solutions. J. Am. Chem. Soc. 2009, 131, 8354-+.

16. Margarella, A. M.; Perrine, K. A.; Lewis, T.; Faubel, M.; Winter, B.; Hemminger, J. C., Dissociation of Sulfuric Acid in Aqueous Solution: Determination of the Photoelectron Spectral Fingerprints of H2so4, Hso4-, and So42- in Water. J. Phys. Chem. C 2013, 117, 8131-8137.

17. Ottosson, N.; Faubel, M.; Bradforth, S. E.; Jungwirth, P.; Winter, B., Photoelectron Spectroscopy of Liquid Water and Aqueous Solution: Electron Effective Attenuation Lengths and Emission-Angle Anisotropy.J. Electron. Spectrosc. 2010, 177, 60-70. 
18. Perrine, K. A.; Van Spyk, M. H. C.; Margarella, A. M.; Winter, B.; Faubel, M.; Bluhm, H.; Hemminger, J. C., Characterization of the Acetonitrile Aqueous Solution/Vapor Interface by Liquid-Jet X-Ray Photoelectron Spectroscopy. J. Phys. Chem. C 2014, 118, 29378-29388.

19. Winter, B.; Faubel, M.; Vacha, R.; Jungwirth, P., Behavior of Hydroxide at the Water/Vapor Interface. Chem. Phys. Lett. 2009, 474, 241-247.

20. Winter, B.; Weber, R.; Schmidt, P. M.; Hertel, I. V.; Faubel, M.; Vrbka, L.; Jungwirth, P., Molecular Structure of Surface-Active Salt Solutions: Photoelectron Spectroscopy and Molecular Dynamics Simulations of Aqueous Tetrabutylammonium Iodide. J. Phys. Chem. B 2004, 108, 14558-14564.

21. Ghosal, S.; Hemminger, J. C.; Bluhm, H.; Mun, B. S.; Hebenstreit, E. L. D.; Ketteler, G.; Ogletree, D. F.; Requejo, F. G.; Salmeron, M., Electron Spectroscopy of Aqueous Solution Interfaces Reveals Surface Enhancement of Halides. Science 2005, 307, 563566.

22. Langmuir, I., Vapor Pressures, Evaporation, Condensation and Adsorption. J. Am. Chem. Soc. 1932, 54, 2798-2832.

23. Onorato, R. M.; Otten, D. E.; Saykally, R. J., Adsorption of Thiocyanate Ions to the Dodecanol/Water Interface Characterized by Uv Second Harmonic Generation. Proc. Natl. Acad. Sci. U.S.A. 2009, 106, 15176-15180.

24. Gonella, G.; Lutgebaucks, C.; de Beer, A. G. F.; Roke, S., Second Harmonic and Sum-Frequency Generation from Aqueous Interfaces Is Modulated by Interference. J. Phys. Chem. C 2016, 120, 9165-9173.

25. Thurmer, S.; Seidel, R.; Faubel, M.; Eberhardt, W.; Hemminger, J. C.; Bradforth, S. E.; Winter, B., Photoelectron Angular Distributions from Liquid Water: Effects of Electron Scattering. Phys. Rev. Lett. 2013, 111, 5.

26. Olivieri, G.; Parry, K. M.; Powell, C. J.; Tobias, D. J.; Brown, M. A., Quantitative Interpretation of Molecular Dynamics Simulations for X-Ray Photoelectron Spectroscopy of Aqueous Solutions. J. Chem. Phys. 2016, 144.

27. Tarbuck, T. L.; Richmond, G. L., Adsorption of Organosulfur Species at Aqueous Surfaces: Molecular Bonding and Orientation. J. Phys. Chem. B 2005, 109, 2086820877.

28. Lewis, T.; Winter, B.; Stern, A. C.; Baer, M. D.; Mundy, C. J.; Tobias, D. J.; Hemminger, J. C., Dissociation of Strong Acid Revisited: X-Ray Photoelectron Spectroscopy and Molecular Dynamics Simulations of Hnoz in Water. J. Chem. Phys. B 2011, 115, 9445-9451.

29. Lewis, T.; Winter, B.; Stern, A. C.; Baer, M. D.; Mundy, C. J.; Tobias, D. J.; Hemminger, J. C., Does Nitric Acid Dissociate at the Aqueous Solution Surface? J. Chem. Phys. C 2011, 115, 21183-21190.

30. Unger, I.; Seidel, R.; Thurmer, S.; Pohl, M. N.; Aziz, E. F.; Cederbaum, L. S.; Muchova, E.; Slavicek, P.; Winter, B.; Kryzhevoi, N. V., Observation of ElectronTransfer-Mediated Decay in Aqueous Solution. Nat. Chem. 2017, 9, 708-714.

31. Thurmer, S.; Oncak, M.; Ottosson, N.; Seidel, R.; Hergenhahn, U.; Bradforth, S. E.; Slavicek, P.; Winter, B., On the Nature and Origin of Dicationic, Charge-Separated Species Formed in Liquid Water on X-Ray Irradiation. Nat. Chem. 2013, 5, 590-596. 
32. Pohl, M. N.; Richter, C.; Lugovoy, E.; Seidel, R.; Slavicek, P.; Aziz, E. F.; Abel, B.; Winter, B.; Hergenhahn, U., Sensitivity of Electron Transfer Mediated Decay to Ion Pairing. J. Chem. Phys. B 2017, 121, 7709-7714.

33. Ellison, G. B.; Tuck, A. F.; Vaida, V., Atmospheric Processing of Organic Aerosols. J. Geophys. Res.-Atmos. 1999, 104, 11633-11641.

34. Bates, T. S.; Lamb, B. K.; Guenther, A.; Dignon, J.; Stoiber, R. E., Sulfur Emissions to the Atmosphere from Natural Sources. J. Atmos. Chem. 1992, 14, 315-337.

35. Lovelock, J. E.; Maggs, R. J.; Rasmusse.Ra, Atmospheric Dimethyl Sulfide and Natural Sulfur Cycle. Nature 1972, 237, 452-\&.

36. Yin, F. D.; Grosjean, D.; Seinfeld, J. H., Photooxidation of Dimethyl Sulfide and Dimethyl Disulfide .1. Mechanism Development. J. Atmos. Chem. 1990, 11, 309-364.

37. Enami, S.; Sakamoto, Y.; Hara, K.; Osada, K.; Hoffmann, M. R.; Colussi, A. J., "Sizing" Heterogeneous Chemistry in the Conversion of Gaseous Dimethyl Sulfide to Atmospheric Particles. Environ. Sci. Technol. 2016, 50, 1834-1843.

38. Shantz, N. C.; Leaitch, W. R.; Caffrey, P. F., Effect of Organics of Low Solubility on the Growth Rate of Cloud Droplets. J. Geophys. Res.-Atmos. 2003, 108.

39. Allen, H. C.; Gragson, D. E.; Richmond, G. L., Molecular Structure and Adsorption of Dimethyl Sulfoxide at the Surface of Aqueous Solutions. J. Phys. Chem. B 1999, 103, 660-666.

40. Chen, X. K.; Minofar, B.; Jungwirth, P.; Allen, H. C., Interfacial Molecular Organization at Aqueous Solution Surfaces of Atmospherically Relevant Dimethyl Sulfoxide and Methanesulfonic Acid Using Sum Frequency Spectroscopy and Molecular Dynamics Simulation. J. Phys. Chem. B 2010, 114, 15546-15553.

41. Winter, B., Liquid Microjet for Photoelectron Spectroscopy. Nucl. Instrum. Meth. A 2009, 601, 139-150.

42. Seidel, R.; Pohl, M. N.; Ali, H.; Winter, B.; Aziz, E. F., Advances in Liquid Phase Soft-X-Ray Photoemission Spectroscopy: A New Experimental Setup at BESSY II. Rev. Sci. Instrum. 2017, 88.

43. Kurahashi, N.; Karashima, S.; Tang, Y.; Horio, T.; Abulimiti, B.; Suzuki, Y.-I.; Ogi, Y.; Oura, M.; Suzuki, T., Photoelectron Spectroscopy of Aqueous Solutions: Streaming Potentials of $\operatorname{Nax}(\mathrm{X}=\mathrm{Cl}, \mathrm{Br}$, and I) Solutions and Electron Binding Energies of Liquid Water and X. J. Chem. Phys. 2014, 140.

44. Kuo, I. F. W.; Mundy, C. J., An Ab Initio Molecular Dynamics Study of the Aqueous Liquid-Vapor Interface. Science 2004, 303, 658-660.

45. Mundy, C. J.; Kuo, I. F. W., First-Principles Approaches to the Structure and Reactivity of Atmospherically Relevant Aqueous Interfaces. Chem. Rev. 2006, 106, 1282-1304.

46. Phillips, J. C.; Braun, R.; Wang, W.; Gumbart, J.; Tajkhorshid, E.; Villa, E.; Chipot, C.; Skeel, R. D.; Kale, L.; Schulten, K., Scalable Molecular Dynamics with Namd. J. Comput. Chem. 2005, 26, 1781-1802.

47. MacKerell, A. D., et al., All-Atom Empirical Potential for Molecular Modeling and Dynamics Studies of Proteins. J. Chem. Phys. B 1998, 102, 3586-3616.

48. Strader, M. L.; Feller, S. E., A Flexible All-Atom Model of Dimethyl Sulfoxide for Molecular Dynamics Simulations. J. Phys. Chem. A 2002, 106, 1074-1080.

49. Klauda, J. B.; Venable, R. M.; Freites, J. A.; O'Connor, J. W.; Tobias, D. J.; Mondragon-Ramirez, C.; Vorobyov, I.; MacKerell, A. D.; Pastor, R. W., Update of the 
Charmm All-Atom Additive Force Field for Lipids: Validation on Six Lipid Types. J. Phys. Chem. B 2010, 114, 7830-7843.

50. Jorgensen, W. L.; Chandrasekhar, J.; Madura, J. D.; Impey, R. W.; Klein, M. L., Comparison of Simple Potential Functions for Simulating Liquid Water. J. Chem. Phys. 1983, 79, 926-935.

51. Grubmuller, H.; Heller, H.; Windemuth, A.; Schulten, K., Generalized Verlet Algorithm for Efficient Molecular Dynamics Simulations with Long-Range Interactions. Mol. Simulat. 1991, 6, 121-142.

52. Ryckaert, J. P.; Ciccotti, G.; Berendsen, H. J. C., Numerical-Integration of Cartesian Equations of Motion of a System with Constraints - Molecular-Dynamics of N-Alkanes. J. Comput. Phys. 1977, 23, 327-341.

53. Miyamoto, S.; Kollman, P. A., Settle - an Analytical Version of the Shake and Rattle Algorithm for Rigid Water Models. J. Comput. Chem. 1992, 13, 952-962.

54. Essmann, U.; Perera, L.; Berkowitz, M. L.; Darden, T.; Lee, H.; Pedersen, L. G., A Smooth Particle Mesh Ewald Method. J. Chem. Phys. 1995, 103, 8577-8593.

55. Martyna, G. J.; Tobias, D. J.; Klein, M. L., Constant-Pressure Molecular-Dynamics Algorithms. J. Chem. Phys. 1994, 101, 4177-4189.

56. Feller, S. E.; Zhang, Y. H.; Pastor, R. W.; Brooks, B. R., Constant-Pressure Molecular-Dynamics Simulation - the Langevin Piston Method. J. Chem. Phys. 1995, 103, 4613-4621.

57. Humphrey, W.; Dalke, A.; Schulten, K., Vmd: Visual Molecular Dynamics. J. Mol. Graph. Model. 1996, 14, 33-38.

58. Winter, B.; Weber, R.; Widdra, W.; Dittmar, M.; Faubel, M.; Hertel, I. V., Full Valence Band Photoemission from Liquid Water Using Euv Synchrotron Radiation. J. Phys. Chem. A 2004, 108, 2625-2632.

59. Pinto, R.; Ramanathan, K. V.; Babu, R. S., Reactive Ion Etching in Sf6 GasMixtures. J. Elec. Soc. 1987, 134, 165-175.

60. Compton, R. N.; Huebner, R. H.; Reinhardt, P. W.; Christophorou, L. G., Threshold Electron Impact Excitation of Atoms and Molecules - Detection of Triplet and Temporary Negative Ion States. J. Chem. Phys. 1968, 48, 901-+.

61. Francis, J. T.; Turci, C. C.; Tyliszczak, T.; Desouza, G. G. B.; Kosugi, N.; Hitchcock, A. P., Electron-Impact Core Excitation of Sf6 .1. S-2p, S-2s, and F-1s Spectroscopy. Phys. Rev. A 1995, 52, 4665-4677.

62. Bjorneholm, O.; Werner, J.; Ottosson, N.; Ohrwall, G.; Ekholm, V.; Winter, B.; Unger, I.; Soderstrom, J., Deeper Insight into Depth-Profiling of Aqueous Solutions Using Photoelectron Spectroscopy. J. Phys. Chem. C 2014, 118, 29333-29339.

63. Jungwirth, P.; Tobias, D. J., Specific Ion Effects at the Air/Water Interface. Chem. Rev. 2006, 106, 1259-1281.

64. Jungwirth, P.; Tobias, D. J., Ions at the Air/Water Interface. J. Phys. Chem. B 2002, 106, 6361-6373.

65. Jungwirth, P.; Tobias, D. J., Molecular Structure of Salt Solutions: A New View of the Interface with Implications for Heterogeneous Atmospheric Chemistry.J. Chem. Phys. B 2001, 105, 10468-10472.

66. Perrine, K. A.; Parry, K. M.; Stern, A. C.; Van Spyk, M. H. C.; Makowski, M. J.; Freites, J. A.; Winter, B.; Tobias, D. J.; Hemminger, J. C., Specific Cation Effects at 
Aqueous Solution-Vapor Interfaces: Surfactant-Like Behavior of Li+ Revealed by Experiments and Simulations. Proc. Natl. Acad. Sci. USA 2017, 114, 13363-13368.

67. Winter, B.; Faubel, M., Photoemission from Liquid Aqueous Solutions. Chem. Rev. 2006, 106, 1176-1211.

68. Cooper, J.; Zare, R. N., Angular Distribution of Photoelectrons. The Journal of Chemical Physics 1968, 48, 942.

69. Schmidt, V., Electron Spectrometry of Atoms Using Synchrotron Radiation. In Electron Spectroscopy of Atoms Using Synchrotron Radiation, University Press: Cambridge, 1997.

70. Turri, G., et al., Probing the Molecular Environment Using Spin-Resolved Photoelectron Spectroscopy. Phys. Rev. Lett. 2004, 92.

71. Turri, G., et al., Spin- and Angle-Resolved Spectroscopy of S 2p Photoionization in the Hydrogen Sulfide Molecule. Phys. Rev. A 2004, 70, 7.

72. Kukk, E.; Bozek, J. D.; Sheehy, J. A.; Langhoff, P. W.; Berrah, N., Angular Distribution of Molecular-Field- and Spin-Orbit-Split Sulfur 2p Photoemission in Ocs: A Sensitive Probe of the Molecular Environment. J. Phys. B-At. Mol. Opt. Phys. 2000, 33, L51-L57.

73. Sander, R., Compilation of Henry's Law Constants (Version 4.0) for Water as Solvent. Atmospheric Chem. Phys. 2015, 15, 4399-4981.

74. Sander, R., Compilation of Henry's Law Constants for Inorganic and Organic Species of Potential Importance in Environmental Chemistry; http://satellite.mpic.de/henry/henry/henry-3.0.pdf.

75. Errington, J. R.; Debenedetti, P. G., Relationship between Structural Order and the Anomalies of Liquid Water. Nature 2001, 409, 318-321.

76. Duboue-Dijon, E.; Laage, D., Characterization of the Local Structure in Liquid Water by Various Order Parameters. J. Phys. Chem. B 2015, 119, 8406-8418.

77. Wu, X. G.; Lu, W. J.; Streacker, L. M.; Ashbaugh, H. S.; Ben-Amotz, D., Temperature-Dependent Hydrophobic Crossover Length Scale and Water Tetrahedral Order. J. Phys. Chem. Lett. 2018, 9, 1012-1017.

78. Farrell, J. R.; Mctigue, P., Precise Compensating Potential Difference Measurements with a Voltaic Cell - the Surface-Potential of Water. J. Electroanal. Chem. 1982, 139, 37-56. 


\section{Figure Captions}

Figure 1 Sulfur 2p photoelectron spectra acquired from aqueous solutions of mixtures of (A) 0.5 M DMSO and 0.5 $\mathrm{M} \mathrm{DMSO}_{2}$, and (B) $0.5 \mathrm{M} \mathrm{DMSO}$ and $0.5 \mathrm{M} \mathrm{DMSO}_{3}$. The photon energy was $260 \mathrm{eV}$. Raw data points are indicated by black circles, (fitted) signal contributions from DMSO, $\mathrm{DMSO}_{2}$, and $\mathrm{DMSO}_{3}$ are highlighted by individual Gaussians (blue, orange, and green, respectively - for clarity all Gaussians are also shown by dashed grey lines), and the total fit is shown as a solid red line.

Figure 2 Cartoon of two possible surface arrangements of the DMSO and $\mathrm{DMSO}_{2}$ aqueous mixtures that could lead to the intensity distributions observed in Figure 1A; layered (top) and smoothly mixed (bottom). The curves to the right indicate qualitative density profiles of DMSO (blue) and $\mathrm{DMSO}_{2}$ (green) in the two cases as a function of distance from the surface. The PAD analysis (Figures 4-5) indicate that the bottom cartoon best describes the mixed solutions, as indicated by the red box.

Figure 3 Sulfur 2p photoelectron spectrum acquired from $0.2 \mathrm{M} \mathrm{DMS}$, using $260 \mathrm{eV}$ photon energy. The solid red line indicates the total fit to the measured data (black dots).

Figure 4 Sulfur 2p photoelectron spectra from an aqueous solution mixture of DMSO (1.0 $\mathrm{M})$ and $\mathrm{DMSO}_{2}(1.0 \mathrm{M})$ measured at $310 \mathrm{eV}$ photon energy. Spectra are shown for three electron detection angles: $0^{\circ}, 54.7^{\circ}$ (magic angle), and $90^{\circ}$ with respect to the light polarization vector. The inset shows the same spectra but scaled to the same intensity at maximum signal.

Figure 5 Integrated signal intensities of the S 2p photoelectron spectrum recorded for the same $1 \mathrm{M}$ DMSO / $1 \mathrm{M} \mathrm{DMSO}_{2}$ solution as in Figure 3. The photon energy was $310 \mathrm{eV}$ (main figure). The error bars are estimated from experimental reproducibility. The solid line shows a fit to Eq. 1 (see text). The inset shows fitted $\beta_{2}$-values as a function of kinetic energy of the $S 2 p$ photoelectrons (recorded at $310 \mathrm{eV}, 400 \mathrm{eV}$, and $640 \mathrm{eV}$ photon energies); all $\beta_{2}$-values were obtained as indicated in the main figure. 
Figure 6 Representative MD snapshots from (A) nominal 0.25 M DMS, and (B) $0.25 \mathrm{M}$ DMSO slab calculations, and density profiles of (C) DMS S (black) and water O atoms (blue) and (D) DMS S (black) and water $\mathrm{O}$ atoms (blue). The coloring scheme in the snapshots is: $\mathrm{C}$ atoms, silver, S atoms, yellow, DMSO O atoms, red; DMS and DMSO H atoms, white; water O atoms, blue; water $\mathrm{H}$ atoms, light gray.

Figure 7 Radial distribution functions (RDFs), $g(r)$, of water $\mathrm{O}$ atoms around (A) the $\mathrm{S}$ atom of DMS, (B) the S atom of DMSO, and (C) the central C atom (C2) of propane in bulk water. In (A) and (B). In panels (A) and (B) the RDFs for DMS and DMSO in the bulk are plotted as solid curves and the RDFs of interfacial DMS and DMSO at the aqueous interface are plotted as dashed lines.

Figure 8 Density isosurfaces of water $\mathrm{O}$ atoms around the $\mathrm{S}$ atoms of DMS (left panel), DMSO (middle panel), and the central $\mathrm{C}$ atom of propane (right panel). The contour levels are $0.041 \AA^{-3}$ for DMS and $0.044 \AA^{-3}$ for DMSO and propane.

Figure 9 Histograms of tetrahedral order parameters averaged over the first solvation shell for water molecules solvating water $\mathrm{O}$ atoms (blue), the $\mathrm{S}$ atom in DMS (black), the $\mathrm{S}$ atom in DMSO (red), and the central $\left(\mathrm{C}_{2}\right)$ atom in propane (green) in bulk water.

Figure 10 Number of water molecules in the first solvation shell (defined using $4.1 \AA$ cutoff) of DMSO and DMS. The Gibbs dividing surface (GDS) is the location along the surface normal where the water density is equal to half its bulk value. 


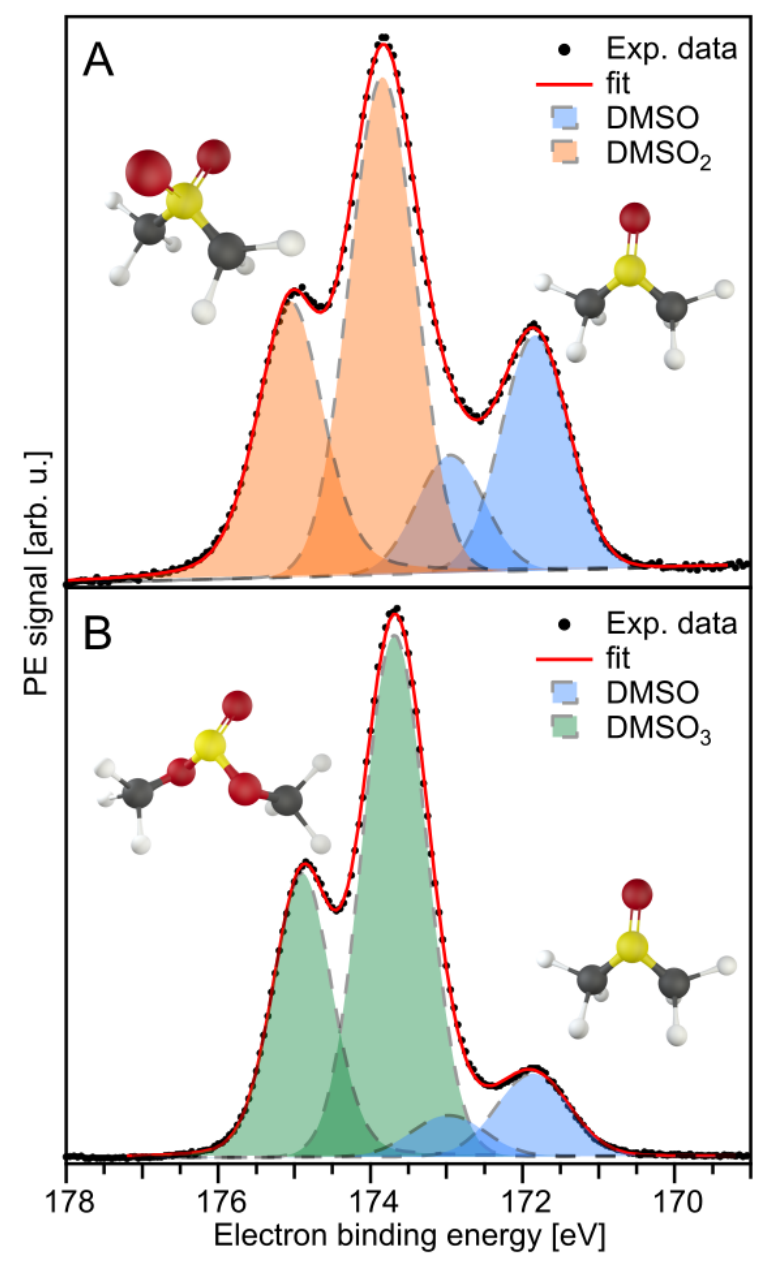

Figure 1 

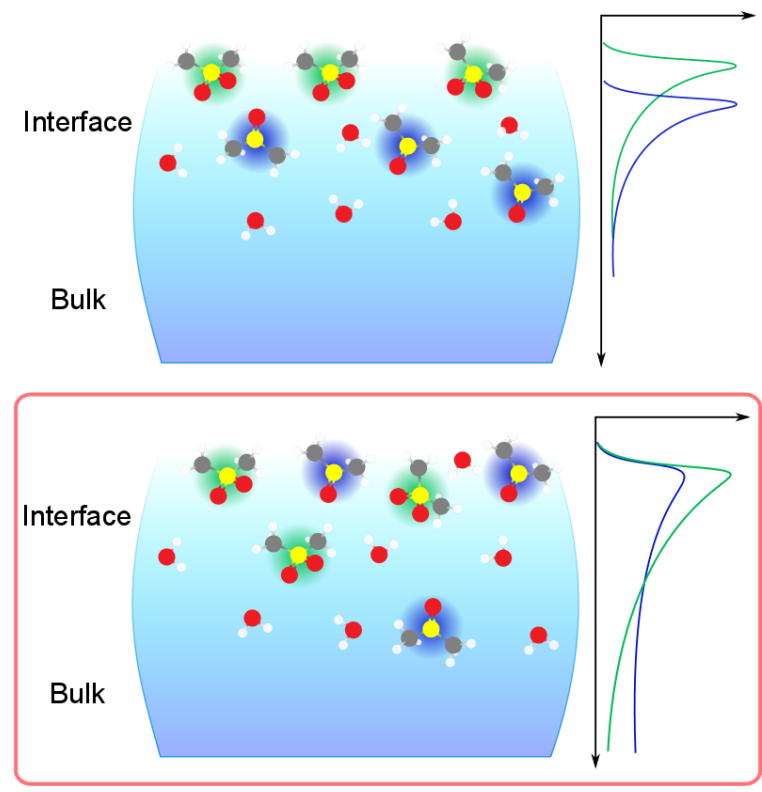

Figure 2

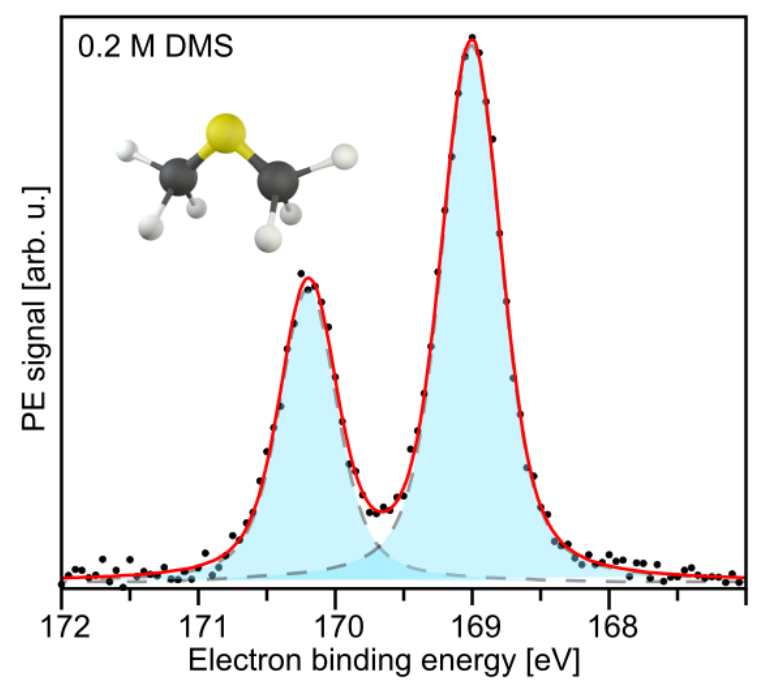

Figure 3 


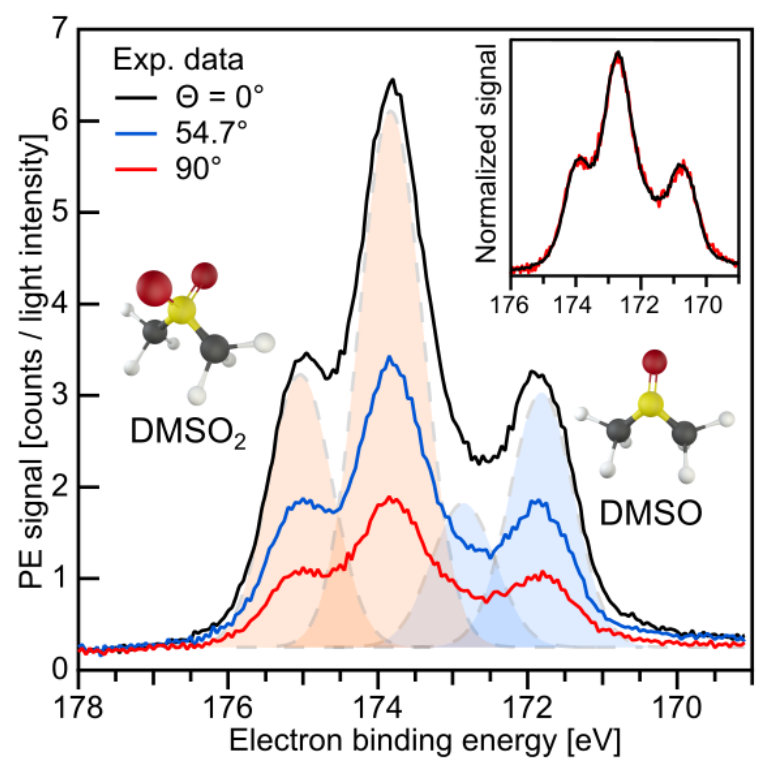

Figure 4

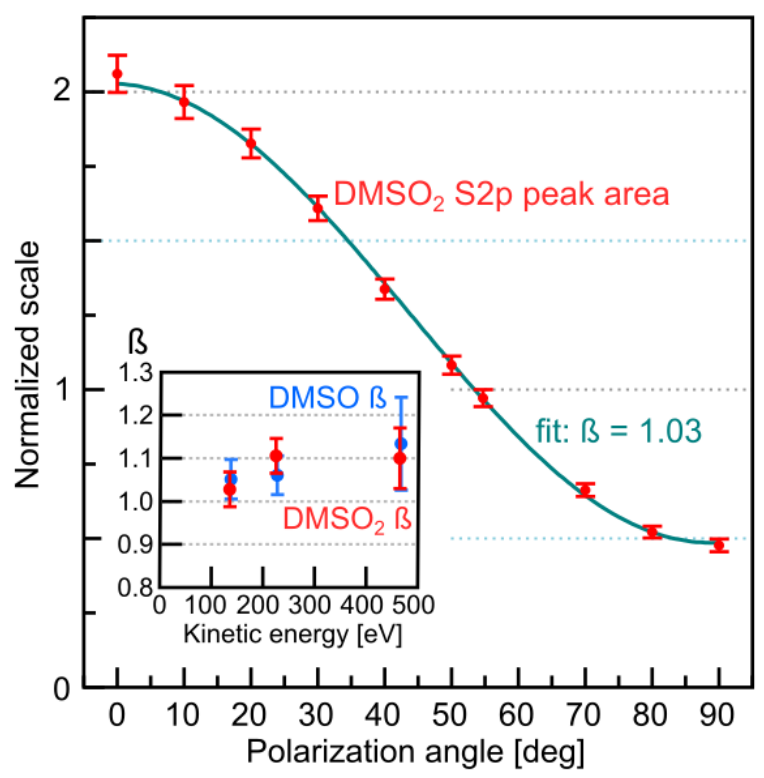

Figure 5 


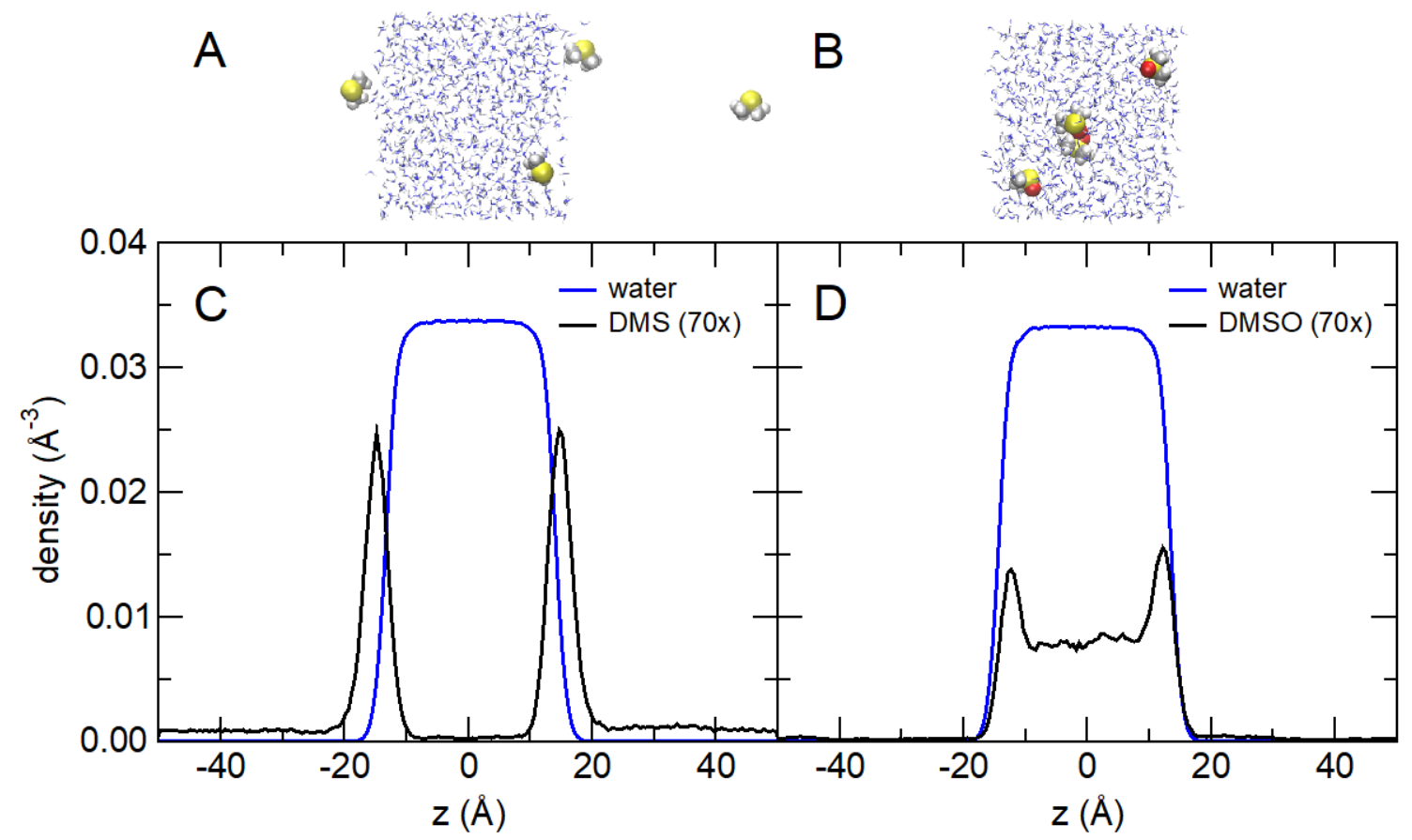

Figure 6

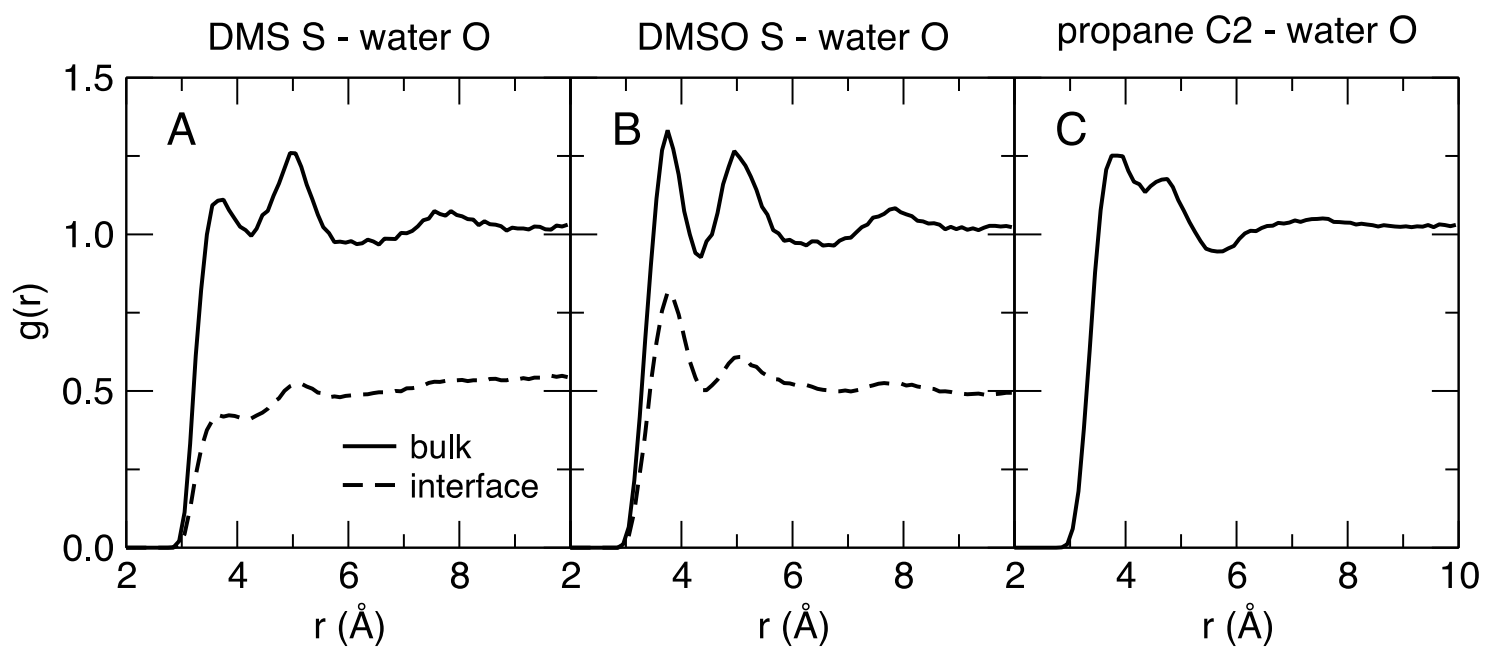

Figure 7 

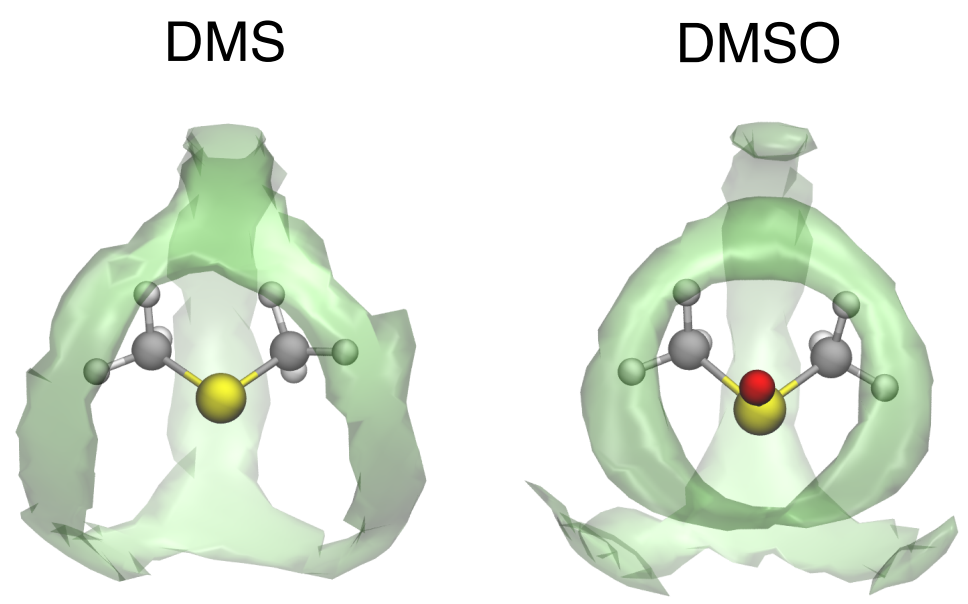

propane

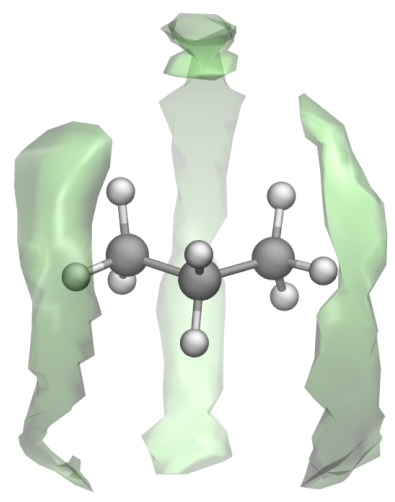

Figure 8

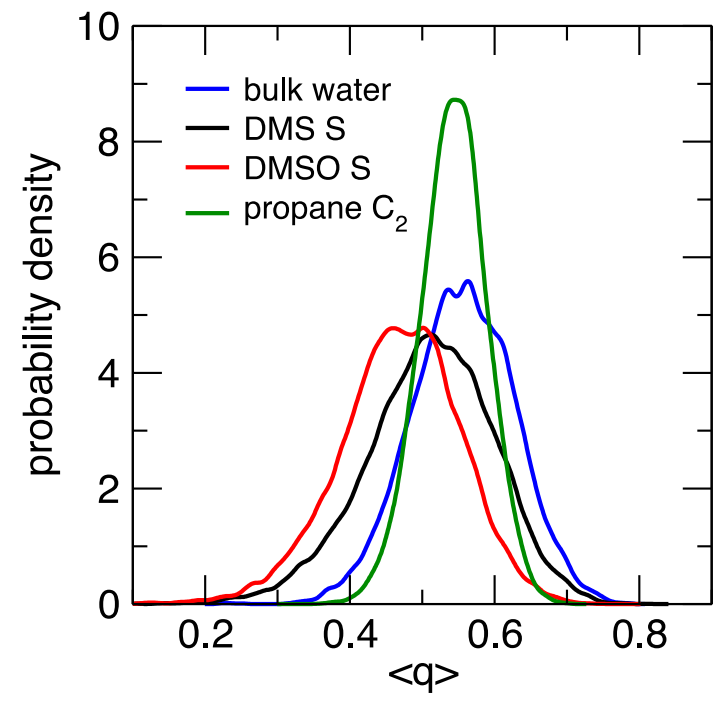

Figure 9 


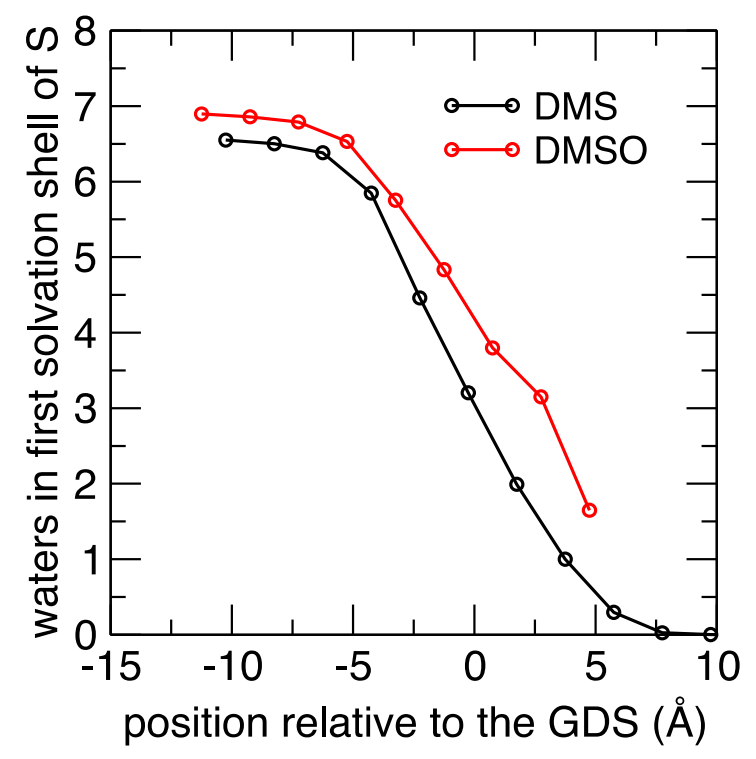

Figure 10 


\section{Table}

Table I. Electron binding energies (eBE) measured for the Sulfur 2p orbital (both spin-orbit states), energy shifts of organosulfur compounds (" $\mathrm{X}$ ") relative to DMS, binding motifs, and oxidation states.

\begin{tabular}{lccccc}
\hline \hline Compound & eBE S P P/2 & eBE S P & $\Delta$ E (X-DMS) & Bonds to O & Oxidation state \\
\hline DMS & $169 \mathrm{eV}$ & $170.1 \mathrm{eV}$ & & 0 & -2 \\
$\mathrm{DMSO}$ & $171.9 \mathrm{eV}$ & $173 \mathrm{eV}$ & $2.9 \mathrm{eV}$ & 2 & 0 \\
$\mathrm{DMSO}_{2}$ & $173.8 \mathrm{eV}$ & $175 \mathrm{eV}$ & $4.8-9 \mathrm{eV}$ & 4 & 2 \\
$\mathrm{DMSO}_{3}$ & $173.8 \mathrm{eV}$ & $175 \mathrm{eV}$ & $4.8-9 \mathrm{eV}$ & 4 & 4 \\
\hline \hline
\end{tabular}

